'División de Anestesiología, Escuela de Medicina, Pontificia Universidad Católica de Chile.

${ }^{2}$ Departamento de Medicina Interna, Escuela de Medicina, Pontificia Universidad Católica de Chile.

${ }^{3}$ Escuela de Enfermería Universidad FinisTerrae. Chile.

${ }^{4}$ División de Cirugía, Escuela de Medicina, Pontificia Universidad Católica de Chile. aEnfermera Universitaria.

Recibido el 30 de noviembre de 2011, aceptado el 23 de marzo de 2012

Correspondencia a Dra. Marcia Corvetto. Marcoleta 367, tercer piso, División de Anestesiología. Pontificia Universidad Católica de Chile, Santiago, Chile.

Fono 562-23543270

Fax 562-23543270.

E-mail:mcorvett@med.puc.cl

\section{Simulación en educación médica: una sinopsis}

\author{
MARCIA CORVETTO ${ }^{1}$, MARÍA PÍA BRAVO ${ }^{1}$, \\ RODRIGO MONTAÑA' ${ }^{1}$, FRANCO UTILI ${ }^{2}$, ELIANA ESCUDERO ${ }^{3, a}$, \\ CAMILO BOZA $^{4}$, JULIÁN VARAS ${ }^{4}$, JORGE DAGNINO ${ }^{1}$
}

\section{Simulation in medical education: a synopsis}

Clinical simulation is defined as a technique (not a technology) to replace or amplify real experiences with guided experiences that evoke or replicate substantial aspects of the real world in a fully interactive fashion. Over the past few years, there has been a significant growth in its use, both as a learning tool and as an assessment for accreditation. Example of this is the fact that simulation is an integral part of medical education curricula abroad. Some authors have cited it as an unavoidable necessity or as an ethical imperative. In Chile, its formal inclusion in Medical Schools' curricula has just begun. This review is an overview of this important educational tool, presenting the evidence about its usefulness in medical education and describing its current situation in Chile.

(Rev Med Chile 2013; 141: 70-79).

Key words: Education, Medical; Patient Simulation; Teaching.
S imular es representar algo, fingiendo o imitando lo que no es ${ }^{1}$. En el área de la salud, consiste en situar a un estudiante en un contexto que imite algún aspecto de la realidad clínica ${ }^{2}$. Gaba la define como una técnica, no una tecnología, para sustituir o ampliar las experiencias reales a través de experiencias guiadas, que evocan o replican aspectos sustanciales del mundo real, de una forma totalmente interactiva ${ }^{3}$.

En medicina ha sido utilizada para reproducir experiencias reales de pacientes a través de escenarios adecuadamente guiados y controlados ${ }^{4}$. La simulación crea un ambiente ideal para la educación, debido a que las actividades pueden diseñarse para que sean predecibles, consistentes, estandarizadas, seguras y reproducibles ${ }^{5}$.

Hoy en día, la simulación es parte integral del currículo de educación en medicina en otros países $^{6}$. Además, ha pasado a ser parte de las evaluaciones necesarias para obtener la Licencia Médica en Estados Unidos de Norteamérica ${ }^{7,8}$ y para la acreditación de ciertas especialidades médicas 9 .

En nuestro país, a pesar de que muchos grupos la han utilizado desde hace años, su inserción for- mal en los currículos de las Escuelas de Medicina recién comienza. Con el objetivo de dar a conocer a la comunidad médica esta creciente herramienta educacional y dar una visión realista de cómo se ha desarrollado su aplicación en Chile, se presenta esta revisión.

\section{Historia de la simulación clínica}

El grado de desarrollo y diversidad alcanzado por la simulación clínica hace difícil sintetizar su historia sin limitarse a un área específica o etapa de evolución. Habiendo muy buenas revisiones recientes ${ }^{10-13}$, hemos preferido enfocarnos en los factores que han ido impulsando ese desarrollo y en sugerir tres etapas en esta evolución.

En el desarrollo de la simulación clínica moderna, con apenas medio siglo de evolución, es posible discernir la influencia de cuatro fuerzas: 1) El desarrollo de la bioética, desde la declaración de Helsinski en 1964 que protege a los individuos como sujetos de experimentación, hasta la actualidad donde la atención se ha enfocado hacia los derechos de los pacientes ${ }^{14}$; 2) El desarrollo 
de la educación médica, con mayores exigencias para asegurar su calidad y con el cambio desde el paradigma basado en la duración temporal de los procesos a uno centrado en la demostración de competencias objetivables; 3 ) La preocupación creciente por la seguridad de los pacientes como sujetos pasivos en los procesos de educación clínica; 4) El desarrollo tecnológico en computación, electrónica, nuevos materiales, la háptica ${ }^{15}$ y la realidad virtual.

En relación a las etapas de este desarrollo, es posible distinguir tres períodos:

1. Los precursores, desde 1929 hasta la década de los sesenta, marcada por los simuladores de vuelo aunque también se cita otras aplicaciones en el ámbito militar y de la medicina ${ }^{16,17}$.

2. Los pioneros, desde los sesenta a fines de los ochenta, partiendo con Laerdal, Abrahamson, Gravenstein y Gaba, quienes desarrollaron simuladores complejos, capaces de replicar características anatómicas y eventos fisiológi$\cos ^{18-20}$.

3. La consolidación, en las dos últimas décadas, con la aceptación creciente de la simulación como un complemento y a veces como substituto ventajoso de la formación clínica. Se desarrollan maniquíes de mayor sofisticación y a precios más accesibles. Surge también un gran número de simuladores de tareas específicas quirúrgicas, diagnósticas y de procedimientos. Paralelamente, se desarrolla la investigación sobre la utilidad de la simulación clínica en el desarrollo de competencias clínicas; muestra de ello es la expansión exponencial del número de artículos publicados en los últimos diez años.

\section{Simulación en educación médica: tipos, ventajas y aplicaciones}

Existen múltiples clasificaciones en la literatura de las diferentes alternativas de simulación que se utilizan en clínica ${ }^{6,21-25}$. Una de ellas es la descrita por Ziv, que divide las herramientas en 5 categorías principales $^{21}$ :

1. Simuladores de uso específico y de baja tecnología: En inglés part task trainers, son modelos diseñados para replicar sólo una parte del organismo y del ambiente por lo que sólo permiten el desarrollo de habilidades psicomotoras básicas. Por ejemplo, un brazo para punción venosa o una cabeza para intubación traqueal ${ }^{25}$.
2. Pacientes simulados o estandarizados: Actores entrenados para actuar como pacientes. Se utilizan para entrenamiento y evaluación de habilidades en obtención de la historia clínica, realización del examen físico y comunicación ${ }^{26}$.

3. Simuladores virtuales en pantalla: Son programas computacionales que permiten simular diversas situaciones, en áreas como la fisiología, farmacología o problemas clínicos, e interactuar con el o los estudiantes ${ }^{25}$. Su principal objetivo es entrenar y evaluar conocimientos y la toma de decisiones. Una ventaja es que permite el trabajo de varios estudiantes a la vez; de hecho, actualmente hay programas para entrenamiento de trabajo en equipo ${ }^{27}$.

4. Simuladores de tareas complejas: Mediante el uso de modelos y dispositivos electrónicos, computacionales y mecánicos, de alta fidelidad visual, auditiva y táctil se logra una representación tridimensional de un espacio anatómico. Dichos modelos generados por computadores son frecuentemente combinados con part task trainers que permiten la interacción física con el ambiente virtual. Usados para el entrenamiento de tareas complejas, permiten desarrollar habilidades manuales y de orientación tridimensional, adquirir conocimientos teóricos y mejorar la toma de decisiones. Ha sido utilizada ampliamente en cirugía laparoscópica y procedimientos endoscópicos ${ }^{25}$.

5. Simuladores de paciente completo: Maniquíes de tamaño real, manejados computacionalmente que simulan aspectos anatómicos y fisiológicos. Permiten desarrollar competencias en el manejo de situaciones clínicas complejas y para el trabajo en equipo ${ }^{6}$.

Otro punto importante de mencionar es el concepto de fidelidad de los simuladores o de una simulación. Clásicamente se ha utilizado este término para definir el grado de realismo de los modelos y de la experiencia en la que se usan, dividiéndolos en tres niveles ${ }^{25}$ :

1. Simulación de baja fidelidad: Modelos que simulan sólo una parte del organismo, usados generalmente para adquirir habilidades motrices básicas en un procedimiento simple o examen físico; por ejemplo, la instalación de una vía venosa periférica o la auscultación cardiaca básica. 
2. Simulación de fidelidad intermedia: Se combina el uso de una parte anatómica, con programas computacionales de menor complejidad que permiten al instructor manejar variables fisiológicas básicas con el objetivo de lograr el desarrollo de una competencia. Por ejemplo, dispositivos para el entrenamiento de reanimación cardiopulmonar.

3. Simulación de alta fidelidad: Integra múltiples variables fisiológicas para la creación de escenarios clínicos realistas con maniquíes de tamaño real. El fin es entrenar competencias técnicas avanzadas y competencias en el manejo de crisis.

La falta de consistencia en el uso del término fidelidad ha llevado en algunos casos a confusión, ya que se ha utilizado para definir complejidad o tecnología ${ }^{25}$. La fidelidad no es siempre proporcional a la complejidad utilizada. Por ejemplo, cuando se realiza la entrevista medica a un paciente simulado, en el que además se le tiene que examinar el rojo pupilar, se trata de una simulación de muy alta fidelidad ya que se acerca mucho a la realidad, sin embargo, es un escenario de baja complejidad y escasa tecnología.

Las ventajas del uso de la simulación clínica en la educación médica han sido ampliamente des$\operatorname{critas}^{3,25,28}$. Proporciona un ambiente controlado y seguro, que permite crear y reproducir situaciones o escenarios a demanda, permite el entrenamiento sistemático y repetido de habilidades prácticas y competencias, permite equivocarse y aprender del error, el proceso de aprendizaje se basa en la práctica y la reflexión, logrando una mayor transferencia de la formación desde la teoría a la práctica y finalmente nos sirve como herramienta de evaluación ${ }^{28}$. Además, permite el entrenamiento consistente y programado en situaciones clínicas de presentación poco habitual, enfermedades raras y situaciones críticas $^{29}$. Por último, dicho entrenamiento que puede adecuarse individualmente para cada alumno, no conlleva riesgos ni para el alumno ni para el paciente ${ }^{25}$. Gracias a estas ventajas, la medicina la ha utilizado con fines tanto educacionales como evaluativos ${ }^{5}$.

Múltiples son las aplicaciones y metodologías de simulación utilizadas en educación médica y las áreas de entrenamiento que ellas cubren ${ }^{6,21-25}$. Con el objetivo de describir dichas metodologías, qué habilidades se logran con cada una de ellas y el uso habitual que se le da a cada una, hemos utilizado la tipología descrita por Alinier ${ }^{30}$. Esta clasificación describe las herramientas y técnicas educacionales que se utilizan en simulación, agrupándolas en 6 niveles tecnológicos; simulaciones escritas, modelos tridimensionales, simuladores basados en pantallas computacionales, pacientes estandarizados, simuladores de pacientes de fidelidad intermedia y simuladores de pacientes de alta fidelidad (Tabla 1). Estos distintos niveles permiten el desarrollo de distintas habilidades, competencias y performance, de acuerdo a la pirámide descrita por Miller en $1990^{31}$. Es importante mencionar que cuando nos referimos a competencia, implica adquisición de conocimiento, habilidades y destrezas y el concepto de performance significa desempeño o rendimiento ${ }^{32}$.

Por último, como ya se mencionó anteriormente, la simulación también es una valiosa herramienta de evaluación. Se utiliza como metodología de evaluación de habilidades psicomotoras y comunicacionales ${ }^{5}$. También se ha utilizado exitosamente para evaluar el desarrollo de competencias ${ }^{7}$. Gracias a esto, se ha convertido en un método estándar de evaluación en múltiples áreas ${ }^{21,33}$. Ejemplo de esto que la prueba conocida como ECOE (Evaluación Clínica Objetiva Estructurada) o en inglés OSCE (Objective Structured Clinical Examination) se ha convertido en parte esencial en la acreditación de licencias médicas en Canadá y Estados Unidos de Norteamérica $^{34,35}$. El formato básico consiste en un circuito de estaciones secuenciales en el que se utilizan pacientes simulados estandarizados, casos por computador, maniquíes, pruebas complementarias (ECG, RX, analítica, etc.) y preguntas de respuesta múltiple o corta relacionadas con los $\operatorname{casos}^{29}$. Actualmente, los esfuerzos están puestos en identificar y validar las distintas metodologías y escalas de evaluación basadas en simulación?

\section{Evidencia de su utilidad en Medicina}

La educación y el entrenamiento basados en simulación han demostrado su efectividad en múltiples áreas. Específicamente ha demostrado mejorar la adquisición de conocimiento médico, la comunicación y el trabajo en equipo, el desarrollo de ciertas habilidades, disminuir el estrés durante los procedimientos e incluso ha mostrado directa mejoría de ciertos resultados clínicos ${ }^{5}$. 
Tabla 1. Tipos de metodologías de simulación. (Adaptado de Alinier, Medical Teacher, 2007)

\begin{tabular}{|c|c|c|c|c|c|c|}
\hline & Nivel 0 & Nivel 1 & Nivel 2 & Nivel 3 & Nivel 4 & Nivel 5 \\
\hline $\begin{array}{l}\text { Técnica de } \\
\text { simulación }\end{array}$ & $\begin{array}{l}\text { Simulaciones } \\
\text { escritas }\end{array}$ & $\begin{array}{l}\text { Simuladores de } \\
\text { baja fidelidad, } \\
\text { part task } \\
\text { trainers y ma- } \\
\text { niquíes básicos }\end{array}$ & $\begin{array}{l}\text { Simuladores } \\
\text { de pantallas } \\
\text { computaciona- } \\
\text { les, simulado- } \\
\text { res virtuales y } \\
\text { simuladores } \\
\text { quirúrgicos }\end{array}$ & $\begin{array}{l}\text { Pacientes } \\
\text { estandarizados }\end{array}$ & $\begin{array}{l}\text { Simuladores } \\
\text { de fidelidad } \\
\text { intermedia y } \\
\text { maniquíes de } \\
\text { tamaño real } \\
\text { no totalmente } \\
\text { interactivos }\end{array}$ & $\begin{array}{l}\text { Simuladores de } \\
\text { alta fidelidad y } \\
\text { maniquíes de } \\
\text { tamaño real } \\
\text { totalmente } \\
\text { interactivos }\end{array}$ \\
\hline $\begin{array}{l}\text { Habilidades } \\
\text { que se logran }\end{array}$ & $\begin{array}{l}\text { Cognitivas } \\
\text { pasivas }\end{array}$ & Psicomotoras & $\begin{array}{l}\text { Cognitivas } \\
\text { interactivas }\end{array}$ & $\begin{array}{l}\text { Psicomotoras, } \\
\text { cognitivas e } \\
\text { interpersonales }\end{array}$ & $\begin{array}{l}\text { Parcialmente } \\
\text { interactivas, } \\
\text { psicomotoras, } \\
\text { cognitivas e } \\
\text { interpersonales }\end{array}$ & $\begin{array}{l}\text { Interactivas, } \\
\text { psicomotoras, } \\
\text { cognitivas e } \\
\text { interpersonales }\end{array}$ \\
\hline Uso habitual & $\begin{array}{l}\text { Manejo y } \\
\text { diagnóstico de } \\
\text { pacientes } \\
\text { Evaluación }\end{array}$ & $\begin{array}{l}\text { Práctica de } \\
\text { habilidades }\end{array}$ & $\begin{array}{l}\text { Manejo clínico } \\
\text { de habilidades } \\
\text { cognitivas }\end{array}$ & $\begin{array}{l}\text { Igual que } \\
\text { nivel } 2 \\
\text { Realización de } \\
\text { examen físico, } \\
\text { diagnóstico } \\
\text { y manejo de } \\
\text { pacientes }\end{array}$ & $\begin{array}{l}\text { Igual que } \\
\text { nivel } 3 \\
\text { Habilidades en } \\
\text { procedimientos } \\
\text { Entrenamiento } \\
\text { de simulación } \\
\text { "full-scale" }\end{array}$ & $\begin{array}{l}\text { Igual que } \\
\text { nivel } 4\end{array}$ \\
\hline
\end{tabular}

La simulación como herramienta de educación en pregrado se ha usado exitosamente en la enseñanza de ciencias básicas ${ }^{36-39}$, en el entrenamiento del examen físico de pacientes ${ }^{40-43} \mathrm{y}$ en el entrenamiento de habilidades quirúrgicas y de procedimientos como cricotirotomías, punciones venosas periféricas, punciones lumbares e instalación de tubos pleurales ${ }^{44-46}$. Se han observado ventajas como menor estrés y mejor disposición de los alumnos a realizar ciertos procedimientos solos, al ser entrenados antes con simulación ${ }^{45} \mathrm{y}$ también mejor disposición de los pacientes, cuando los alumnos han sido entrenados previamente con simulación ${ }^{46}$.

En el área de postgrado, la simulación también se ha utilizado ampliamente, demostrando mejoras en el desempeño como resultado del entrenamiento basado en simulación en medicina intensiva, medicina de urgencia y pediatría ${ }^{47-50}$. En anestesiología, hay evidencia de que ha mejorado el desempeño tanto de residentes como especialistas en escenarios de alta complejidad ${ }^{51-55}$. Específicamente en relación a la introducción de protocolos de ACLS (Advanced Cardiac Life Support) ha permitido mejorar el trabajo en equipo y el desempeño clínico en relación al cumplimiento de dichos protocolos ${ }^{56}$. En el área quirúrgica, su desarrollo también ha sido amplio, lo cual se ejemplifica fácilmente con la gran cantidad de simuladores quirúrgicos que existen en la actualidad, los cuales van desde entrenadores de tipo cajas hasta simuladores virtuales ${ }^{57}$. Existen datos que avalan el uso de la simulación para el desarrollo de habilidades tanto en laparoscopia como en endoscopia ${ }^{58-61}$. Específicamente en simulación quirúrgica laparoscópica, se ha demostrado que un entrenamiento en base a competencias que incluye simulación virtual y programas básicos como el Fundamentals of Laparoscopic Surgery, permite adquirir habilidades básicas laparoscópicas con buenos resultados en sala operatoria para procedimientos como colecistectomías ${ }^{62-64}$. Sin embargo, aún queda por demostrar que la simulación permite obtener habilidad quirúrgica avanzada y que ésta se transfiere a la sala operatoria.

Por último, en obstetricia también han sido numerosos los aportes de la simulación en la realización de amniocentesis bajo ultrasonografía, el manejo de la distocia de hombro y de emergencias obstétricas y trauma ${ }^{65-70}$. Interesante mención merece un estudio publicado por Draycott, que muestra una reducción de injuria neonatal de $9,3 \%$ a $2,3 \%$, posterior al entrenamiento con un simulador de distocia de hombro ${ }^{70}$.

Finalmente, podemos afirmar que a pesar de todas las ventajas descritas, la evidencia en cuanto a resultados clínicos todavía es escasa, siendo este sin duda, el mayor desafío en investigación. 


\section{Simulación en Chile}

La primera aproximación a la simulación en Chile fue dada por la Pontificia Universidad Católica de Chile el año 2003, con una escuela de actores que simulaban patologías, al ser interrogados por alumnos de medicina. Más formalmente, en el año 2004 el Instituto Duoc UC, creó el primer centro de simulación para la formación de carreras técnicas en salud, con construcción de escenarios y guías de evaluación.

No existe evidencia científica, pero lo que se conoce a través de la información recopilada, es que desde el 2006 en adelante algunas instituciones de Educación Superior comienzan a incorporar simuladores en la actividad docente. Algunos académicos hacen esfuerzos importantes para desarrollar estos recursos en las universidades y están presentes en seminarios como es la Escuela de Medicina de la Universidad Diego Portales con sus trabajos en farmacología. Otras como la Universidad de Chile y la Universidad Católica presentan proyectos educativos al estado (MECESUP) para crear sus propios centros, lo que también se ha seguido realizando hasta la fecha por otras organizaciones. Sin embargo, fue la Universidad de las Américas la que creó el primer centro de alta fidelidad del país con la creación de la carrera de Enfermería el 2008, que se inició con integración de la simulación en el curriculum. Finalmente, es la Universidad de Los Andes la primera Escuela de Medicina de Chile, que desarrolló también en el 2008 un proyecto de integración de la simulación en el curriculum, dando inicio formal al uso de este modelo en el curso correspondiente al Internado en cirugía, en la unidad de trauma.

En la actualidad son varias las escuelas y facultades que han incorporado esta metodología a sus modelos docentes, pero no es aún un estándar de todas las propuestas curriculares. Los antecedentes identifican a la Universidad Andrés Bello, Pontificia Universidad Católica de Chile, Universidad del Bío Bío, Universidad Finis Terrae, Universidad de las Américas, Universidad Diego Portales y Duoc UC con centros de alta fidelidad.

Con el propósito de presentar de forma más detallada que metodología se utiliza en la actualidad en cada uno de los centros en Chile, hemos enviado una encuesta a los coordinadores de simulación de cada centro. Se les hizo saber que el propósito era desarrollar un documento con evidencia de la información existente en relación a la educación con simulación en Chile. En esta encuesta se preguntó información que se detalla a continuación; año de inicio como centro de simulación, metros cuadrados, descripción de áreas existentes, modelos disponibles, carreras que usan el centro en actividades integradas al curriculum o estables en el tiempo, número de profesores capacitados formalmente en simulación, promedio de uso mensual del laboratorio y actividades de extensión y de investigación. Se recibió respuesta del $87 \%$ de los centros encuestados. Dicha información de encuentra resumida en las Tablas 2 y 3.

Por último, queremos mencionar que en noviembre de 2011 se conformó la "Sociedad Chilena de Simulación Clínica y Seguridad del Paciente". Los objetivos propuestos por dicha Sociedad fueron los siguientes:

a) Contribuir a posicionar la simulación clínica como un modelo educativo pedagógico, fundamental en la educación de ciencias de la salud de nuestro país.

b) Velar por el progreso y desarrollo en la investigación tanto para el ámbito clínico como educativo.

c) Ser el ente oficial, colaborador y asesor de organismos que requieran de la asistencia en materias que velen por la calidad de la atención en salud y la seguridad del paciente.

d) Servir de red de comunicación entre las instituciones y profesionales que realicen o quieran realizar simulación clínica.

e) Establecer vínculos con las empresas u organismos nacionales y/o internacionales, que deseen el desarrollo de la simulación clínica en Chile.

\section{El futuro y los desafíos}

La simulación clínica en la educación y formación de profesionales de la salud ha evolucionado de forma importante, pero no ha alcanzado todavía una aceptación generalizada ${ }^{10}$. Su implementación en programas de pregrado y postítulo ha impactado positivamente la educación en diferentes aspectos como la estandarización de la enseñanza, la incorporación de temas no considerados formalmente en los currículos, en la familiarización de los estudiantes con métodos de autoevaluación y autoaprendizaje, en la ética en temas de salud y en un aspecto difícil de tolerar 
Simulación en educación médica - M. Corvetto et al

Tabla 2. Centros de simulación en Chile

\begin{tabular}{|c|c|c|c|c|c|}
\hline Institución & $\begin{array}{l}\text { Centro de } \\
\text { simulación }\end{array}$ & $\begin{array}{l}\text { Año de } \\
\text { inicio }\end{array}$ & $\begin{array}{c}\text { Metros } \\
\text { disponibles }\end{array}$ & Distribución & $\begin{array}{c}\text { Carreras que usan } \\
\text { el centro }\end{array}$ \\
\hline $\begin{array}{l}\text { Universidad } \\
\text { Diego Portales }\end{array}$ & $\begin{array}{l}\text { Centro de Simulación } \\
\text { Clínica de la Facultad } \\
\text { de Medicina }\end{array}$ & 2010 & $400 \mathrm{~m}^{2}$ & $\begin{array}{l}4 \text { salas de simulación } \\
\text { clínica hospitalaria, } \\
1 \text { área de atención } \\
\text { ambulatoria, } 1 \text { sala } \\
\text { espejo, } 1 \text { sala entrena- } \\
\text { miento con simuladores } \\
\text { de tareas }\end{array}$ & $\begin{array}{l}\text { Pregrado Enfermería y } \\
\text { Medicina, Postgrado } \\
\text { Anestesiología }\end{array}$ \\
\hline $\begin{array}{l}\text { Pontificia } \\
\text { Universidad } \\
\text { Católica de Chile }\end{array}$ & $\begin{array}{l}\text { Centro de Simulación } \\
\text { de la Facultad de } \\
\text { Medicina }\end{array}$ & 2010 & $100 \mathrm{~m}^{2}$ & $\begin{array}{l}3 \text { salas multiuso, una } \\
\text { sala de alta fidelidad }\end{array}$ & $\begin{array}{l}\text { Pregrado Medicina y } \\
\text { Odontología. Postgrado } \\
\text { Neurología, Cirugía, } \\
\text { Anestesia y Urgencia }\end{array}$ \\
\hline $\begin{array}{l}\text { Universidad } \\
\text { Andrés Bello }\end{array}$ & $\begin{array}{l}\text { Centro de Simulación } \\
\text { de la Facultad de } \\
\text { Medicina }\end{array}$ & 2010 & $650 \mathrm{~m}^{2}$ & $\begin{array}{l}5 \text { salas de habilidades y } \\
3 \text { salas de alta fidelidad }\end{array}$ & $\begin{array}{l}\text { Pregrado de Medicina } \\
\text { y Enfermería }\end{array}$ \\
\hline $\begin{array}{l}\text { Universidad Finis } \\
\text { Terrae }\end{array}$ & $\begin{array}{l}\text { Centro de Simulación } \\
\text { Clínica }\end{array}$ & 2009 & $150 \mathrm{~m}^{2}$ & $\begin{array}{l}3 \text { salas de habilidades, } \\
2 \text { salas de alta fidelidad, } \\
2 \text { salas de debriefing }\end{array}$ & $\begin{array}{l}\text { Pregrado Enfermería y } \\
\text { Medicina }\end{array}$ \\
\hline $\begin{array}{l}\text { Universidad de } \\
\text { las Américas }\end{array}$ & $\begin{array}{l}6 \text { centros, } 4 \text { en } \mathrm{RM}, 1 \\
\text { en } V \text { región y } 1 \text { en } \mathrm{VIII} \\
\text { región }\end{array}$ & 2008 & $\begin{array}{l}\text { En conjunto } \\
\text { todos los } \\
\text { centros suman } \\
1.000 \mathrm{~m}^{2}\end{array}$ & $\begin{array}{l}\text { Salas de habilidades, } \\
\text { baja y alta fidelidad }\end{array}$ & $\begin{array}{l}\text { Pregrado Enfermería, } \\
\text { Técnicos de nivel } \\
\text { superior en Enfermería, } \\
\text { Kinesiología y Nutrición }\end{array}$ \\
\hline $\begin{array}{l}\text { Universidad } \\
\text { Andrés Bello }\end{array}$ & $\begin{array}{l}\text { Centro de Simulación } \\
\text { de la Facultad de } \\
\text { Enfermería }\end{array}$ & 2011 & $140 \mathrm{~m}^{2}$ & $\begin{array}{l}\text { Sala de habilidades y } \\
\text { sala espejo }\end{array}$ & Pregrado de Enfermería \\
\hline $\begin{array}{l}\text { Universidad de } \\
\text { los Andes }\end{array}$ & $\begin{array}{l}\text { Unidad de Simulación } \\
\text { Escuela de Enfermería }\end{array}$ & 1995 & $50 m^{2}$ & 1 sala & Pregrado Enfermería \\
\hline Duoc & 5 centros & 2004 & $\begin{array}{c}\text { Sin } \\
\text { información }\end{array}$ & Sin información & Sin información \\
\hline
\end{tabular}

en la docencia tradicional como es el usar el error como un medio de aprendizaje ${ }^{71}$.

Las cuatro fuerzas impulsoras seguirán actuando, por lo que, habrá instrumentos y escenarios de simulación cada vez más sofisticados y realistas. Igualmente, es esperable la ampliación de su uso en pre y postgrado para el desarrollo y mantención de competencias en forma sistemática y reproducible, particularmente en el entrenamiento de equipos y en eventos infrecuentes o catastróficos. Otras áreas de desarrollo esperable, es el ensayo previo de procedimientos o cirugías complejas, el desarrollo de nuevas técnicas terapéuticas y diagnósticas y el diseño de nuevo instrumental y equipamiento.

Por último, parece probable su mayor uso y desarrollo en la evaluación de individuos e instituciones con fines de certificación, recertificación y de acreditación ${ }^{72}$. Para esto, el principal desafío es generar más y mejor investigación, que permita validar la simulación en los ámbitos descritos y particularmente saltar desde el laboratorio a mejorar el cuidado de los pacientes ${ }^{73}$. Cumplida la validación, el desafío siguiente será integrar, y no superponer, la simulación en los procesos de formación clínica a lo largo de toda la vida laboral de los individuos ${ }^{3}$. La capacitación docente y la integración curricular darán el sello final a este desafío. En Chile, el desafío es acortar la brecha actual en este desarrollo. Para ello, es esencial la formación de profesores que puedan usar la simulación clínica en lo conceptual y en lo técnico, para lograr los mejores resultados formativos posibles, el desarrollo de investigación que fortalezca ese desarrollo docente y la difusión e intercambio de conocimiento entre todos los actores relevantes. 
Tabla 3. Centros de simulación en Chile

\begin{tabular}{|c|c|c|c|c|c|}
\hline Institución & Modelos & $\begin{array}{l}\text { Tipos de } \\
\text { simula- } \\
\text { ción }\end{array}$ & $\begin{array}{l}\text { n docen- } \\
\text { tes capa- } \\
\text { citados }\end{array}$ & $\begin{array}{l}\text { Utilizan } \\
\text { pautas } \\
\text { de eva- } \\
\text { luación }\end{array}$ & $\begin{array}{l}\text { Actividades } \\
\text { de inves- } \\
\text { tigación y } \\
\text { extensión }\end{array}$ \\
\hline $\begin{array}{l}\text { Universidad } \\
\text { Diego Portales. } \\
\text { Centro de Simulación } \\
\text { Clínica de la Facultad } \\
\text { de Medicina }\end{array}$ & $\begin{array}{l}\text { SimMan, simulador adulto y Noelle } 565 \text {, } \\
\text { simulador materno fetal y neonatal, Me- } \\
\text { gaCode Kid advanced y Nursing Kid con } \\
\text { VitalSim, } 2 \text { Nursing Anne, Resusci Anne, } \\
\text { Resusci Anne Skill guide con Vital Sim y } \\
\text { part task trainers }\end{array}$ & $\begin{array}{l}\text { Habilidades, } \\
\text { baja y alta } \\
\text { fidelidad }\end{array}$ & 5 & Sí & Si ambas \\
\hline $\begin{array}{l}\text { Pontificia Universidad } \\
\text { Católica de Chile. } \\
\text { Centro de Simulación } \\
\text { de la Facultad de } \\
\text { Medicina }\end{array}$ & $\begin{array}{l}\text { SimMan, ALS Baby 200, ALS Trainer, } \\
\text { Nursing Anne con Vital Sim, Resusci } \\
\text { Anne con Skill Reporter, ResusciTorso } \\
\text { para accesos venosos centrales }\end{array}$ & $\begin{array}{l}\text { Habilidades, } \\
\text { baja y alta } \\
\text { fidelidad }\end{array}$ & 34 & Sí & Sí ambas \\
\hline $\begin{array}{l}\text { Universidad } \\
\text { Andrés Bello. } \\
\text { Centro de Simulación } \\
\text { de la Facultad de } \\
\text { Medicina }\end{array}$ & $\begin{array}{l}\text { Noelle, Megacode Kelly, ALS } \\
\text { Sistema de audio video Smots }\end{array}$ & $\begin{array}{l}\text { Habilidades, } \\
\text { baja y alta } \\
\text { fidelidad }\end{array}$ & 12 & Sí & Sí ambas \\
\hline $\begin{array}{l}\text { Universidad } \\
\text { Finis Terrae. } \\
\text { Centro de Simulación } \\
\text { Clínica }\end{array}$ & $\begin{array}{l}3 \text { modelos alta fidelidad, } 11 \text { modelos } \\
\text { cuerpo entero baja y mediana fidelidad } \\
\text { (adulto y pediátrico). Sistema de audio y } \\
\text { video SMOTS }\end{array}$ & $\begin{array}{l}\text { Habilidades, } \\
\text { baja y alta } \\
\text { fidelidad }\end{array}$ & 12 & Sí & Sí ambas \\
\hline $\begin{array}{l}\text { Universidad } \\
\text { de las Américas }\end{array}$ & $\begin{array}{l}\text { SimMan, Simbaby, Noelle, Nursing Anne, } \\
\text { Nursing Baby, Virtual IV, múltiples mode- } \\
\text { los para habilidades y destrezas }\end{array}$ & $\begin{array}{l}\text { Habilidades, } \\
\text { baja y alta } \\
\text { fidelidad }\end{array}$ & 40 & Sí & No describen \\
\hline $\begin{array}{l}\text { Universidad } \\
\text { Andrés Bello. } \\
\text { Centro de Simulación } \\
\text { de la Facultad de } \\
\text { Enfermería }\end{array}$ & $\begin{array}{l}1 \text { Nursing Anne, } 1 \text { Resusci Anne, } \\
2 \text { Nursing Baby, } 2 \text { Nursing Kid, } \\
3 \text { Nursing Kelly }\end{array}$ & $\begin{array}{l}\text { Habilidades y } \\
\text { baja } \\
\text { fidelidad }\end{array}$ & 3 & Sí & No describen \\
\hline $\begin{array}{l}\text { Universidad } \\
\text { de los Andes. } \\
\text { Unidad de Simu- } \\
\text { lación Escuela de } \\
\text { Enfermería }\end{array}$ & $\begin{array}{l}\text { Nursing Anne, Modelos RCP adultos, } \\
\text { Modelos recién nacidos niño y niña, } \\
\text { Pelvis de sondeo vesical, modelo de niño } \\
\text { para RCP, brazos de punción arterial y } \\
\text { venosa }\end{array}$ & $\begin{array}{l}\text { Habilidades } \\
\text { y baja } \\
\text { fidelidad }\end{array}$ & $\begin{array}{l}\text { No deta- } \\
\text { Ilan }\end{array}$ & Sí & No describen \\
\hline Duoc & Sin información & $\begin{array}{l}\text { Sin informa- } \\
\text { ción }\end{array}$ & $\begin{array}{l}\text { Sin infor- } \\
\text { mación }\end{array}$ & $\begin{array}{l}\text { Sin infor- } \\
\text { mación }\end{array}$ & $\begin{array}{c}\text { Sin informa- } \\
\text { ción }\end{array}$ \\
\hline
\end{tabular}

Agradecimientos: Agradecemos el aporte de información de los Centros de Simulación en Chile a: Soledad Armijo de la Universidad Diego Portales, Eliana Escudero de la Universidad Finis Terrae, Patricia Gazmuri de la Universidad Andrés Bello, Cristián Jara de Hospitalia, Claudia Morales de la Universidad Finis Terrae, Luis Oliva Peña de la Universidad Andrés Bello, Constanza Sanhueza de la Universidad de los Andes, Franco Utili de la Pontificia Universidad Católica de Chile y Mónica Valenzuela Vidal de la Universidad de las Américas.

\section{Referencias}

1. http://buscon.rae.es/drael/SrvltConsulta?TIPO_BUS=3 \&LEMA=simular.

2. Gaba DM. Improving anesthesiologists' performance by simulating reality. Anesthesiology 1992; 76 (4): 491-4.

3. Gaba DM. The future vision of simulation in health care. Qual Saf Health Care 2004; 13 Suppl 1: i2-10.

4. Mata GV. Las simulaciones en Educación Medica. Educ Med 2007; 10 (3): 147-8.

5. Okuda Y, Bryson EO, DeMaria S Jr, Jacobson L, Quino- 
nes J, Shen B, et al. The utility of simulation in medical education: what is the evidence? Mt Sinai J Med 2009; 76 (4): 330-43.

6. Lane J, Slavin S, Ziv A. Simulation in medical education: A review. Simulation \& Gaming 2001; 32 (3): 297-314.

7. Boulet JR, Murray DJ. Simulation-based assessment in anesthesiology: requirements for practical implementation. Anesthesiology 2010; 112 (4): 1041-52.

8. Dillon GF, Boulet JR, Hawkins RE, Swanson DB. Simulations in the United States Medical Licensing Examination (USMLE). Quality and Safety in Health Care 2004; 13 (suppl 1): i41-i5.

9. Berkenstadt H, Ziv A, Gafni N, Sidi A. Incorporating simulation-based objective structured clinical examination into the Israeli National Board Examination in Anesthesiology. Anesth Analg 2006; 102 (3): 853-8.

10. Cooper JB, Taqueti VR. A brief history of the development of mannequin simulators for clinical education and training. Qual Saf Health Care 2004; 13 Suppl 1: i11-8.

11. Bradley P. The history of simulation in medical education and possible future directions. Med Educ 2006; 40 (3): 254-62.

12. Rosen KR. The history of medical simulation. J Crit Care. 2008 Jun; 23 (2): 157-66.

13. Satava RM. Historical review of surgical simulation--a personal perspective. World J Surg. 2008 Feb; 32 (2): 141-8.

14. Martensen R. The history of bioethics: an essay review. J Hist Med Allied Sci 2001; 56 (2): 168-75.

15. Stone RJ. Haptic feedback: A brief history from telepresence to virtual reality. Lect Notes Comput Sc 2001; 2058: 1-16.

16. Flanagan JC. The critical incident technique. Psychol Bull 1954; 51 (4): 327-58.

17. Safar P, Brown TC, Holtey WJ, Wilder RJ. Ventilation and circulation with closed-chest cardiac massage in man. Jama 1961; 176: 574-6.

18. Gordon MS. Cardiology patient simulator. Development of an animated manikin to teach cardiovascular disease. Am J Cardiol 1974; 34 (3): 350-5.

19. Gaba DM, DeAnda A. A comprehensive anesthesia simulation environment: re-creating the operating room for research and training. Anesthesiology 1988; 69 (3): 387-94.

20. Abramson S DJ, Wolff R. . Effectiveness of a simulator in training anestesiology residents. J Med Educ 1969 (44): 515-9.

21. Ziv A, Wolpe PR, Small SD, Glick S. Simulation-based medical education: an ethical imperative. Acad Med 2003; 78 (8): 783-8.

22. http://jeffbauerphd.com/TEWSMedicalSimulation.pdf.
23. J.G López LVS. Simulación, herramienta para la educación médica. Salud Uninorte 2007; 23 (01): 79-95.

24. Kneebone R. Simulation in surgical training: educational issues and practical implications. Med Educ 2003; 37 (3): 267-77.

25. Maran NJ, Glavin RJ. Low- to high-fidelity simulation - a continuum of medical education? Med Educ 2003; 37 Suppl 1: 22-8.

26. Levine AI, Swartz MH. Standardized patients: the "other" simulation. J Crit Care 2008; 23 (2): 179-84.

27. http://simcenter.duke.edu/3DiTeams.html.

28. Gaba DM. Anaesthesiology as a model for patient safety in health care. BMJ 2000; 320 (7237): 785-8.

29. http://escuela.med.puc.cl/publ/arsmedica/ArsMedica15/ Simulacion.html.

30. Alinier G. A typology of educationally focused medical simulation tools. Med Teach 2007; 29(8): e243-50.

31. Miller GE. The assessment of clinical skills/competence/ performance. Acad Med 1990; 65 (9 Suppl): S63-7.

32. Frank JR, Snell LS, Cate OT, Holmboe ES, Carraccio C, Swing SR, et al. Competency-based medical education: theory to practice. Med Teach 2010; 32 (8): 638-45.

33. Ziv A, Rubin O, Sidi A, Berkenstadt H. Credentialing and certifying with simulation. Anesthesiol Clin 2007; 25 (2): 261-9.

34. Sutnick AI, Stillman PL, Norcini JJ, Friedman M, Regan MB, Williams RG, et al. ECFMG assessment of clinical competence of graduates of foreign medical schools. Educational Commission for Foreign Medical Graduates. Jama 1993; 270 (9): 1041-5.

35. Reznick RK, Blackmore D, Dauphinee WD, Rothman AI, Smee S. Large-scale high-stakes testing with an OSCE: report from the Medical Council of Canada. Acad Med 1996; 71 (1 Suppl): S19-21.

36. Kelsey R, Botello M, Millard B, Zimmerman J. An online heart simulator for augmenting first-year medical and dental education. Proc AMIA Symp 2002; 370-4.

37. Via DK, Kyle RR, Trask JD, Shields CH, Mongan PD. Using high-fidelity patient simulation and an advanced distance education network to teach pharmacology to second-year medical students. J Clin Anesth 2004; 16 (2): 144-51.

38. Seybert AL, Kobulinsky LR, McKaveney TP. Human patient simulation in a pharmacotherapy course. Am J Pharm Educ 2008; 72 (2): 37.

39. Fitch MT. Using high-fidelity emergency simulation with large groups of preclinical medical students in a basic science course. Med Teach 2007; 29 (2-3): 261-3.

40. Okuda Y, Quinones J. The use of simulation in the education of emergency care providers for cardiac emergencies. Int J Emerg Med 2008; 1 (2): 73-7. 
41. Ewy GA, Felner JM, Juul D, Mayer JW, Sajid AW, Waugh RA. Test of a cardiology patient simulator with students in fourth-year electives. J Med Educ 1987; 62 (9): 73843.

42. Issenberg SB, Gordon MS, Greber AA. Bedside cardiology skills training for the osteopathic internist using simulation technology. J Am Osteopath Assoc 2003; 103 (12): 603-7.

43. Issenberg SB, McGaghie WC, Gordon DL, Symes S, Petrusa ER, Hart IR, et al. Effectiveness of a cardiology review course for internal medicine residents using simulation technology and deliberate practice. Teach Learn Med 2002; 14 (4): 223-8.

44. Van Sickle KR, Ritter EM, Smith CD. The pretrained novice: using simulation-based training to improve learning in the operating room. Surg Innov 2006; 13 (3): 198-204.

45. Sánchez LD, Delapena J, Kelly SP, Ban K, Pini R, Perna AM. Procedure lab used to improve confidence in the performance of rarely performed procedures. Eur J Emerg Med 2006; 13 (1): 29-31.

46. Graber MA, Wyatt C, Kasparek L, Xu Y. Does simulator training for medical students change patient opinions and attitudes toward medical student procedures in the emergency department? Acad Emerg Med 2005; 12 (7): 635-9.

47. Fritz PZ, Gray T, Flanagan B. Review of mannequinbased high-fidelity simulation in emergency medicine. Emerg Med Australas 2008; 20 (1): 1-9.

48. Halamek LP, Kaegi DM, Gaba DM, Sowb YA, Smith BC, Smith BE, et al. Time for a new paradigm in pediatric medical education: teaching neonatal resuscitation in a simulated delivery room environment. Pediatrics 2000; 106 (4): E45.

49. Hunt EA, Shilkofski NA, Stavroudis TA, Nelson KL. Simulation: translation to improved team performance. Anesthesiol Clin 2007; 25 (2): 301-19.

50. Britt RC, Reed SF, Britt LD. Central line simulation: a new training algorithm. Am Surg 2007;73 (7): 680-2; discussion 2-3.

51. Schwid HA, Rooke GA, Michalowski P, Ross BK. Screenbased anesthesia simulation with debriefing improves performance in a mannequin-based anesthesia simulator. Teach Learn Med 2001; 13 (2): 92-6.

52. Gaba DM, DeAnda A. The response of anesthesia trainees to simulated critical incidents. Anesth Analg 1989; 68 (4): 444-51.

53. DeAnda A, Gaba DM. Unplanned incidents during comprehensive anesthesia simulation. Anesth Analg 1990; 71 (1): 77-82.

54. Schwid HA, O’Donnell D. Anesthesiologists' manage- ment of simulated critical incidents. Anesthesiology 1992; 76 (4): 495-501.

55. Chopra V, Gesink BJ, de Jong J, Bovill JG, Spierdijk J, Brand R. Does training on an anaesthesia simulator lead to improvement in performance? Br J Anaesth 1994; 73 (3): 293-7.

56. Nishisaki A, Keren R, Nadkarni V. Does simulation improve patient safety? Self-efficacy, competence, operational performance, and patient safety. Anesthesiol Clin 2007; 25 (2): 225-36.

57. Halvorsen FH, Elle OJ, Fosse E. Simulators in surgery. Minim Invasive Ther Allied Technol 2005; 14 (4): 214-23.

58. Dunkin B, Adrales GL, Apelgren K, Mellinger JD. Surgical simulation: a current review. Surg Endosc 2007; 21 (3): 357-66.

59. Sedlack RE, Baron TH, Downing SM, Schwartz AJ. Validation of a colonoscopy simulation model for skills assessment. Am J Gastroenterol 2007; 102 (1): 64-74.

60. Stefanidis D, Korndorffer JR Jr, Sierra R, Touchard C, Dunne JB, Scott DJ. Skill retention following proficiency-based laparoscopic simulator training. Surgery 2005; 138 (2): 165-70.

61. Aggarwal R, Ward J, Balasundaram I, Sains P, Athanasiou T, Darzi A. Proving the effectiveness of virtual reality simulation for training in laparoscopic surgery. Ann Surg 2007; 246 (5): 771-9.

62. Buyske J. The role of simulation in certification. Surgical Clinics of North America 2010; 90 (3): 619-21.

63. Oropesa I, Sánchez-González P, Lamata P, Chmarra MK, Pagador JB, Sánchez-Margallo JA, et al. Methods and tools for objective assessment of psychomotor skills in laparoscopic surgery. J Surg Res 2011; 171 (1): e81-95.

64. van Dongen KW, Ahlberg G, Bonavina L, Carter FJ, Grantcharov TP, Hyltander A, et al. European consensus on a competency-based virtual reality training program for basic endoscopic surgical psychomotor skills. Surg Endosc 2011; 25 (1): 166-71.

65. Gardner R, Raemer DB. Simulation in obstetrics and gynecology. Obstetrics and gynecology clinics of North America. [10.1016/j.ogc.2007.12.008]. 2008; 35 (18319131): 97-127, ix.

66. Maher JE, Kleinman GE, Lile W, Tolaymat L, Steele D, Bernard J. The construction and utility of an amniocentesis trainer. Am J Obstet Gynecol 1998; 179 (5): 1225-7.

67. Dupuis O, Moreau R, Silveira R, Pham MT, Zentner A, Cucherat M, et al. A new obstetric forceps for the training of junior doctors: a comparison of the spatial dispersion of forceps blade trajectories between junior and senior obstetricians. Am J Obstet Gynecol 2006; 194 (6): 1524-31.

68. Deering S, Poggi S, Macedonia C, Gherman R, Satin AJ. 
Simulación en educación médica - M. Corvetto et al

Improving resident competency in the management of shoulder dystocia with simulation training. Obstet Gynecol 2004; 103 (6): 1224-8.

69. Crofts JF, Bartlett C, Ellis D, Hunt LP, Fox R, Draycott TJ. Management of shoulder dystocia: skill retention 6 and 12 months after training. Obstet Gynecol 2007; 110 (5): 1069-74.

70. Draycott TJ, Crofts JF, Ash JP, Wilson LV, Yard E, Sibanda $\mathrm{T}$, et al. Improving neonatal outcome through practical shoulder dystocia training. Obstet Gynecol 2008; 112
(1): 14-20.

71. Utili F. Simulación en el aprendizaje, práctica y certificación de las competencias en medicina. Ars Médica Revista de Estudios Médicos Humanísticos 2007; 15: 197-210.

72. Petrusa ER. Current challenges and future opportunities for simulation in high-stakes assessment. Simul Healthc 2009; 4 (1): 3-5.

73. Gaba DM. Where do we come from? What are we? Where are we going? Simul Healthc 2011; 6 (4): 195-6. 